\title{
ELECTRICAL INVESTIGATION OF THE WATER ADSORPTION MECHANISM ON NATURAL CLINOPTILOLITE
}

\author{
Created by: Gianfranco Carotenuto \\ Version received: 12 March 2020
}

check for

updates

\begin{abstract}
Zeolites are ionic conductors and the cation electrical mobility in zeolites depends on their hydration state; consequently, the water adsorption/desorption process can be simply investigated by measuring the temporal evolution of current intensity in samples exposed to an environment with constant humidity or dry air, respectively. According to this kinetic analysis, a mechanism has been formulated for the water adsorption process able to justify the Lagergren pseudo-first-order kinetics observed for adsorption and the first-order kinetics observed for desorption. In this mechanism water molecules are first attract by the electric field of the cations and then they move at cation-framework interface to maximize the hydrogen bond interactions.
\end{abstract}

\section{Introduction}

Zeolites are microporous silico-alluminate compounds with a regular arrangement of channel\$1]. Such microporosity makes zeolites able to adsorb small molecules like water, ethanol, formaldehyde, acetic acid, etc. [2] Extra-framework charge balancing cations should be involved in the mechanism of gas-phase molecular adsorption in zeolites because long-range electrostatic interactions are possible between the electric field of cations and the total dipole-moment of the adsorbate molecules ${ }^{[3]}$. Such attractive cation effect should act with both polarizable and high polar molecules like, for example, water. However, it is still not clear the exact structure of the hydrated cationic sites and the molecular pathway involved in the hydration process. Such informations can be obtained only by a kinetic analysis of the water adsorption/desorption process, and this aspect has not been deeply investigated yet. Owing to the importance of zeolite adsorption properties, some kinetic studies on the liquid-phase adsorption of zeolites (e.g., for ammonium cations) are available in the literature ${ }^{[1]}$, however to the best of our knowledge very limited is the literature available for gas-phase adsorption of zeolites[4].

Here, an electrical method has been developed to investigate the kinetics of water adsorption/desorption on natural clinoptilolite and a mechanism for these two processes has been formulated on the basis of the experimentally found kinetic orders.

\section{Kinetic analysis method}

The presence of cations in zeolites allows electrical conduction ${ }^{[5]}$. In fact, zeolites are ionic conductors and their conductivity strictly depends on the aluminum content. The cation amount in zeolites increases with decreasing of the $\mathrm{Si} / \mathrm{Al}$ ratio. Therefore, cations have at same time a leading role in physical adsorption of polar/polarizable molecules and electrical transport, and consequently it is possible to investigate the adsorption process (for example, for water) by a simple electrical technique. When a D.C. electric field, E, is applied to a zeolite sample, two different phenomena take place: (i) electrical transport and (ii) electrode polarization. In these conditions, the current intensity, I, is not directly proportional to the charge carrier density (cations concentration in the solid sample), since during the conduction process, cations progressively accumulate at electrode surface and the number of charge carriers decreases. When an A.C. electric field is applied, the amount of cations that stop at the electrode surface decreases significantly, the higher is the frequency. If the A.C. electric field frequency is higher than $1 \mathrm{kHz}$, the amount of cations stopped at electrode surface is almost negligible. Therefore, for a sample biased by a high-frequency A.C. electric field (e.g., $5 \mathrm{kHz}$ ), the current intensity moving in the sample is directly proportional to the charge carrier density (I=z.e.n.v.E, where $\mathbf{z}$ is the cation valence, $\mathrm{e}$ is the elemental charge, $\mathrm{n}$ is the charge carrier density, $\mathrm{v}$ is the cation average speed, and $E$ is the applied local electric field) ${ }^{[6]}$ and the temporal evolution of the normalized current intensity, $\mathrm{I} / \mathrm{I}_{0}$, describes exactly the cation concentration behavior with time. In this case, a kinetic analysis of the adsorption/desorption can be performed by simple current intensity measurements. In particular, owing to the strong 
electrostatic interaction between cations and framework nucleophilic areas, dehydrated cations could be considered as not mobile $(\mu=0)$, while they become mobile after adsorption of one water molecule. Consequently, the temporal evolution of the normalized current intensity, $\mathrm{I} / \mathrm{I}_{0}$, could be used to establish the relative cation concentration increase/decrease, during the process of adsorption/desorption, respectively. Based on this type of investigation, the kinetic order of the water adsorption/desorption process can be established and the kinetic rates measured too.

\section{Results}

The kinetics of the water adsorption process on natural clinoptilolite, studied in isothermal conditions $\left(25^{\circ} \mathrm{C}\right)$, and in presence of a constant humidity environment (75\%), has been found to follow a pseudo-first order behavior 7$]$ (i.e., Lagergren model: $\log \left(\mathbf{1}-Q_{t} / Q_{e}\right)=-k \cdot t / 2.303$, where $Q_{t}$ represents the adsorbed amount of water at time and $Q$ the adsorbed water amount at equilibrium; the $Q_{t} / Q_{e}$ ratio numerically corresponds to the $/ / I_{e}$ ratio) (see Figure $1 A$ ). Differently, a first-order kinetic behavior ${ }^{[8]}$ (i.e., $\log \left(Q_{t} / Q_{0}\right)=-k^{\prime} \cdot \mathbf{t} / \mathbf{2 . 3 0 3}$, where $Q_{t}$ is the adsorbed water amount at $t$ time and $Q_{0}$ is the initial adsorbed amount of water; the $Q_{0} Q_{0}$ ratio numerically corresponds to ${ }_{t} / I_{0}$ ) has been found to control water desorption in dry air (see Figure $1 \mathrm{~B}$ ). A correlation factor, $\mathrm{R}^{2}$, very close to one was found in the bestfittings of data based on these models, thus indicating the validity of each model. The kinetic constants measured for the adsorption and desorption processes are: $\mathrm{k}=0.00586 \mathrm{~min}^{-1}$ and $\mathrm{k}^{\prime}=0.0203 \mathrm{~min}^{-1}$ (at $25^{\circ} \mathrm{C}$ ), respectively. The firstorder kinetic process experimentally found for dehydration exactly corresponds to the inverse of the pseudo-first order hydration process and thus confirms it.
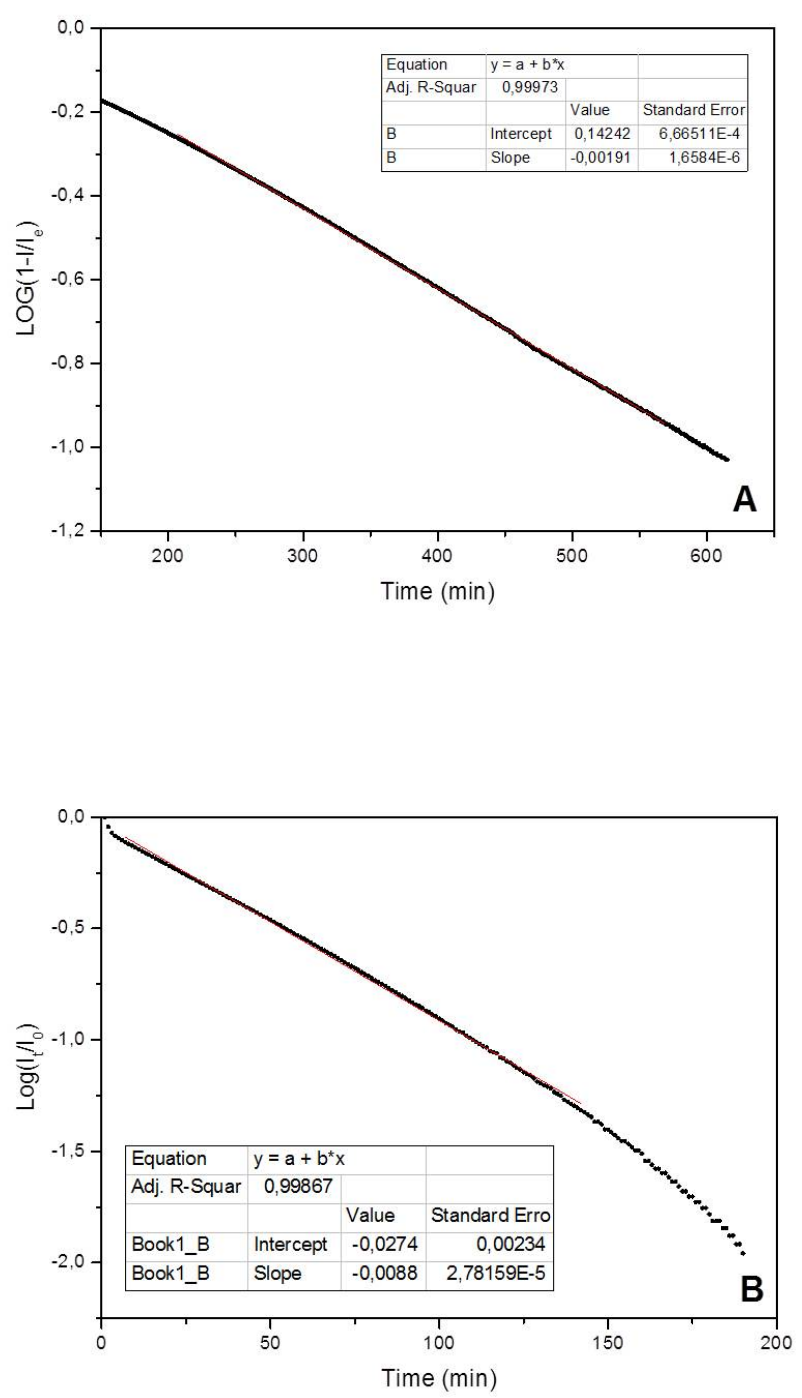
Fig. 1 - Best-fitting of the adsorption/desorption experimental data, obtained by the electrical method.

Such experimentally found kinetic orders suggest an adsorption mechanism based on a direct water-cation electrostatic interaction, with formation of a dipole-cation bond as rate-limiting elemental step (i.e., $\mathrm{F}^{-} \mathrm{Me}^{+}+\mathrm{H}_{2} \mathrm{O} \rightarrow$ $\mathrm{F}^{-} \mathrm{Me}\left(\mathrm{OH}_{2}\right)^{+}$, where $\mathrm{F}^{-}$represents the framework nucleophilic area). However, the significant change of cation mobility, as a consequence of the adsorption process, would suggest the existence of a second, faster elemental step, based on the displacement of the adsorbed water molecule at the framework-cation interface to maximize the hydrogen-bond interactions (i.e., $\left.\mathrm{F}^{-} \mathrm{Me}\left(\mathrm{OH}_{2}\right)^{+} \rightarrow \mathrm{F}^{-} \mathrm{H}_{2} \mathrm{OMe}^{+}\right)$. In fact, the separation of the cation from the negative charge in the framework, as a consequence of the $\mathrm{H}_{2} \mathrm{O}$ molecule interposition, decreases the intensity of the Coulomb's force, making possible cation movement by hopping.

\section{Conclusions}

A kinetic analysis of the physical adsorption/desorption process of water on natural clinoptilolite has been performed by monitoring the temporal evolution of the relative current intensity in a biased sample (sinusoidal voltage of $20 \mathrm{~V}_{\mathrm{pp}}$ and $5 \mathrm{kHz})$, exposed to a constant humidity environment $(75 \%)$ at $25^{\circ} \mathrm{C}$. According to the performed kinetic analysis the adsorption mechanism involves two-steps: slow water-cation electrostatic association, and fast water transfer at framework-cation interface.

\section{Experimental details}

A natural clinoptilolite sample (T.I.P., Germany), in form of slab (5X5X5 mm), was biased by a sinusoidal voltage of $20 V_{p p}(5 \mathrm{kHz})$, provided by a DDS function generator (GW Instek, SFG-1013) and the effective current intensity (dff) was measured by a true-RMS ammeter contained in a $100 \mathrm{kHz}$ bandwidth digital multimeter (Brymen, BM869s). Timeresolved data were recorded on a PC by using the DMM datalogging system. During the hydration/dehydration process, the applied voltage resulted practically a constant because of the very low conductivity of the clinoptilolite sample (the power of the used function generator was of ca. $40 \mathrm{~W}$ ). Silver paint was used for electrical contacts. To achieve an atmosphere with constant humidity, the saturated salt method ${ }^{[9]}$ was used. In particular, the sample was placed inside a sealed container, and a $\mathrm{NaCl} / \mathrm{H}_{2} \mathrm{O}$ system was used to achieve a $75 \%$ humidity atmosphere. Owing to the strict dependence of zeolite conductivity on temperature, all tests were carried out at constant temperature $\left(25^{\circ} \mathrm{C}\right)$. The clinoptilolite slabs were obtained by cutting the raw stone by a small diamond saw (mini drill).

\section{References}

1. Mohau Moshoeshoe, Misael Silas Nadiye-Tabbiruka, Veronica Obuseng; A Review of the Chemistry, Structure, Properties and Applications of Zeolites. American Journal of Materials Science 2017, 7, 196-221, 10.5923/j.materials.20170705.12.

2. Amin Kalantarifard; Jo Gwang Gon; Go-Su Yang; Formaldehyde Adsorption into Clinoptilolite Zeolite Modified with the Addition of Rich Materials and Desorption Performance Using Microwave Heating. Terrestrial, Atmospheric and Oceanic Sciences 2016, 27, 865-875, 10.3319/tao.2016.05.28.01(tt).

3. Xiaowen Xu; Jing Wang; Ying-Cai Long; Zeolite-based Materials for Gas Sensors. Sensors 2006, 6, 1751-1764, 10.3390/s6121751.

4. Gianfranco Carotenuto; Isothermal Kinetic Investigation of the Water-Cations Interaction in Natural Clinoptilolite. European Journal of Engineering Research and Science 2019, 4, 119-125, 10.24018/ejers.2019.4.5.1341.

5. G. Kelemen; G. Schön; Ionic conductivity in dehydrated zeolites. Journal of Materials Science 1992, 27, 6036-6040, 10.1007/bf01133746.

6. Gabriele Kelemen; Wolfgang Lortz; Ionic conductivity of synthetic analcime, sodalite and offretite. Journal of Materials Science 1989, 24, 333-338, 10.1007/bf00660976.

7. Ho Yuh-Shan; Citation review of Lagergren kinetic rate equation on adsorption reactions. Scientometrics 2004, 59, 171-177, 10.1023/b:scie.0000013305.99473.cf.

8. Rumeysa Tekin; Nurcan Bac; Juliusz Warzywoda; A. Sacco; Encapsulation of a fragrance molecule in zeolite X. Microporous and Mesoporous Materials 2015, 215, 51-57, 10.1016/j.micromeso.2015.05.020. 
9. Lewis Greenspan; Humidity fixed points of binary saturated aqueous solutions. Journal of Research of the National Bureau of Standards Section A: Physics and Chemistry 1977, 81, 89, 10.6028/jres.081a.011.

\section{Keywords}

zeolite; clinoptilolite; adsorption; desorption; kinetics; electrical method

c) (i) (C) 2020 by the author(s). Distribute under a Creative Commans CC BY license 